Pacific Journal of Mathematic 


\title{
COMPLEMENTATION PROBLEMS FOR THE BAIRE CLASSES
}

\author{
William G. BADE
}

This paper initiates a study of the classes of Baire measurable functions on the unit interval $I$ from the standpoint of the theory of spaces of continuous functions. For each countable ordinal $\alpha$, the $\alpha$ th Baire class $\mathfrak{B}_{\alpha}$ has a representation as $C\left(\Omega_{\alpha}\right)$, where $\Omega_{\alpha}$ is a certain compactification of the discrete set $I$. For $1 \leqq \alpha<\beta, \mathfrak{B}_{\alpha}$ is a closed subalgebra of $\mathfrak{B}_{\beta}$. The principal result proved here is the fact that $\mathfrak{B}_{\alpha}$ is always uncomplemented as a closed subspace of $\mathfrak{B}_{\beta}$. The method of proof relies on a detailed analysis on the canonical onto map $\phi: \Omega_{\beta} \rightarrow \Omega_{\alpha}$ induced by the imbedding of $\mathfrak{B}_{\alpha}$ in $\mathfrak{B}_{\beta}$, and consists of showing that this map admits no "averaging operator." It depends heavily on recent results in the theory of averaging operators due to S.Z. Ditor.

In this paper scalars and functions are real valued. However, the arguments extend easily to the complex case. In the last section we show how corresponding results may be obtained when $I$ is replaced by any uncountable compact metric space.

1. The Baire classes as function algebras. We shall start by recalling classical definitions and facts concerning the Baire classes of functions on the unit interval $I$. Let $C(I)$ be the class of all real continuous functions on $I$ with supremum norm. Denote by $\mathfrak{B}_{1}$ the class of all bounded functions which are pointwise limits of sequences of functions in $C(I)$, and for each countable ordinal $\alpha$ inductively define $\mathfrak{B}_{\alpha}$ to be the class of all bounded functions on $I$ which are pointwise limits of sequences of functions in $\bigcup_{\beta<\alpha} \mathfrak{B}_{\beta}$. We call $\mathfrak{B}_{\alpha}$ the class of Baire functions of order $\alpha$.

There is another approach to $\mathfrak{B}_{\alpha}$. Each countable ordinal $\alpha$ is even or odd as follows: 1 is odd and each limit ordinal is even; the immediate successor of an even ordinal is odd, and of an odd ordinal is even. Let $F_{0}$ be the class of all closed subsets of $I$; and $F_{1}$ be the class of countable unions of sets in $F_{0}$. For each $\alpha$

(i) $F_{\alpha}$ is the class of all countable unions of sets in $\mathrm{U}_{\beta<\alpha} F_{\beta}$, if $\alpha$ is odd;

(ii) $F_{\alpha}$ is the class of all countable intersections of sets in $\bigcup_{\beta<\alpha} F_{\beta}$, if $\alpha$ is even.

Correspondingly, let $G_{0}$ be the class of all open subsets of $I$, and $G_{1}$ be all countable intersections of $G_{0}$ sets. For each $\alpha$

(iii) $G_{\alpha}$ is the class of all countable intersections of sets in $\bigcup_{\beta<\alpha} G_{\beta}$, 
if $\alpha$ is odd;

(iv) $G_{\alpha}$ is the class of all countable unions of sets in $\bigcup_{\beta<\alpha} G_{\beta}$, if $\alpha$ is even.

If $\alpha$ is odd (even) the class $F_{\alpha}\left(G_{\alpha}\right)$ is called the additive class of type $\alpha$.

If $\alpha$ is odd (even) the class $G_{\alpha}\left(F_{\alpha}\right)$ is called the multiplicative class of type $\alpha$.

For each $\alpha$ define $H_{\alpha}=F_{\alpha} \cap G_{\alpha}$. We call $H_{\alpha}$ the class of ambiguous sets of type $\alpha$.

The classes $F_{\alpha}, G_{\alpha}, H_{\alpha}$ satisfy many simple relationships. In particular, the complement of an $F_{\alpha}$ set is in $G_{\alpha}$, etc., and $H_{\alpha}$ is a field of sets (i.e., closed under finite unions and intersections and complements). For a complete discussion see [4] and [6].

Now for each $\alpha$ denote by $\mathfrak{2}_{\alpha}$ the class of all bounded functions $f$ on $I$ such that for every real $\lambda$, the sets

$$
\{t \mid f(t) \leqq \lambda\},\{t \mid f(t) \geqq \lambda\}
$$

all belong to the multiplicative class of type $\alpha$. The following classical result ([6], page 393) connects $\mathfrak{B}_{\alpha}$ and $\mathfrak{N}_{\alpha}$.

Theorem 1.1. (Lebesgue-Hausdorff). If $\alpha$ is a finite ordinal, $\mathfrak{B}_{\alpha}=\mathfrak{U}_{\alpha}$. If $\alpha$ is infinite, then $\mathfrak{B}_{\alpha}=\mathfrak{A}_{\alpha+1}$.

We apply this theorem to characterize those subsets $A$ of $I$ whose characteristic functions $k_{A}$ are in $\mathfrak{B}_{\alpha}$. Observe that $k_{A} \in \mathfrak{U}_{\alpha}$ iff $A \in H_{\alpha}$.

Corollary 1.2. Let $A$ be a subset of $I$. Then

(a) $k_{A} \in \mathfrak{B}_{\alpha}$ iff $A \in H_{\alpha}$, if $\alpha$ is finite.

(b) $k_{A} \in \mathfrak{B}_{\alpha}$ iff $A \in H_{\alpha+1}$, if $\alpha$ is infinite.

To avoid constantly considering cases we also define the class $K_{\alpha}=H_{\alpha}$ if $\alpha$ is finite and $K_{\alpha}=H_{\alpha+1}$ if $\alpha$ is infinite.

In what follows we shall need a separation theorem ([6], page $351)$.

Lemma 1.3. Let $R$ and $S$ be disjoint subsets of $I$ which are of multiplicative type $\alpha$. There exist disjoint sets $A$ and $B$ belonging to $H_{\alpha}$ such that

$$
R \sqsubseteq A, \quad S \subseteq B
$$

Returning to $\mathfrak{B}_{\alpha}$, we note that it is an algebra of bounded functions on $I$ which is closed under uniform convergence ([4], page 134). Thus $\mathfrak{B}_{\alpha}$ is a Banach algebra of bounded functions under the uniform norm satisfying the obvious condition. 


$$
\left\|f^{2}+g^{2}\right\| \geqq\left\|f^{2}\right\|, f, g \in \mathfrak{B}_{\alpha} \text {. }
$$

Let $\Omega_{\alpha}$ be the compact set of nonzero multiplicative linear functionals on $\mathfrak{B}_{\alpha}$ with the weak star topology. Define

$$
\hat{f}(\omega)=\omega(f), \omega \in \Omega_{\alpha}, f \in \mathfrak{B}_{\alpha} .
$$

By the real form of the Gelfand-Naimark theorem (Arens [1]), the map $\chi: f \rightarrow \hat{f}$ is an isometric algebra isomorphism of $\mathfrak{B}_{\alpha}$ onto $C\left(\Omega_{\alpha}\right)$. Since evaluation at a point of $I$ is multiplicative, there is a natural imbedding $\tau_{\alpha}: I \rightarrow \Omega_{\alpha}$ of $I$ into $\Omega_{\alpha}$ determined by

$$
\hat{f}\left(\tau_{\alpha}(t)\right)=f(t), t \in I, f \in \mathfrak{B}_{\alpha} .
$$

Moreover, $\tau_{\alpha}(I)$ is dense in $\Omega_{\alpha}$, since if $\hat{f}$ vanishes on $\tau_{\alpha}(I), f$ must vanish on $I$. Also $\tau_{\alpha}(I)$ is discrete in its relative topology as a subset of $\Omega_{\alpha}$, since the characteristic function of a point of $I$ belongs to $\mathfrak{B}_{\alpha}$. Thus we may regard $\Omega_{\alpha}$ as a certain compactification of the discrete unit interval.

Our next aim is to prove that $\Omega_{\alpha}$ is totally disconnected. Note that the following statements are equivalent for a function $f \in \mathfrak{B}_{\alpha}$.

(a) $f$ takes only the values 0 and 1 on $I$,

(b) $f=k_{A}$ where $A \in K_{\alpha}$,

(c) $\hat{f}$ takes only the values 0 and 1 on $\Omega_{\alpha}$,

(d) $\hat{f}=k_{B}$ where $B$ is an open and closed subset of $\Omega_{\alpha}$. Moreover, we have

$$
B=\overline{\tau_{\alpha}(A)}, A=\tau_{\alpha}^{-1}\left(B \cap \tau_{\alpha}(I)\right) .
$$

The correspondence $A \rightarrow B$ defines an isomorphism of the Boolean algebra $K_{\alpha}$ of subsets of $I$ onto the Boolean algebra $K^{\alpha}$ of all closed and open subsets of $\Omega_{\alpha}$. To prove that $\Omega_{\alpha}$ is totally disconnected we must prove that $K^{\alpha}$ separates points of $\Omega_{\alpha}$.

THEOREM 1.4. The space $\Omega_{\alpha}$ is totally disconnected.

Proof. Let $\omega_{1}$ and $\omega_{2}$ be distinct points of $\Omega_{\alpha}$. We can find $\hat{f} \in C\left(\Omega_{\alpha}\right)$ such that the sets

$$
U_{1}=\{\omega \mid \hat{f}(\omega) \geqq 1\}, U_{2}=\{\omega \mid \hat{f}(\omega) \leqq 0\}
$$

are closed neighborhoods of $\omega_{1}$ and $\omega_{2}$.

Define $f(t)=\hat{f}\left(\tau_{\alpha}(t)\right), t \in I$. Then the sets $A_{i}=\tau_{\alpha}^{-1}\left(U_{i} \cap \tau(I)\right), i=1,2$ satisfy

$$
A_{1}=\{t \mid f(t) \geqq 1\}, A_{2}=\{t \mid f(t) \leqq 0\},
$$


and thus are of multiplicative class $\alpha(\alpha+1)$ if $\alpha$ is finite (infinite). By Lemma 1.3 there exist disjoint sets $W_{1}, W_{2}$ in $K_{\alpha}$ such that $A_{i} \subseteq W_{i}$. The sets $V_{i}=\overline{\tau_{\alpha}\left(W_{i}\right)}$ are open and closed neighborhoods of $\omega_{i}$. They are disjoint, since otherwise their intersection contains a point of the dense set $\tau_{\alpha}(I)$, contradicting the fact that $W_{1} \cap W_{2}=\varnothing$.

2. Topological tools. In this section we collect some basic facts concerning spaces of continuous functions and projections onto subalgebras. Let $S$ and $T$ be compact Hausdorff spaces and $\phi: S \rightarrow T$ be a continuous onto map. We call the elements of the collection

$$
\Re=\left\{\phi^{-1}(t) \mid t \in T\right\}
$$

fibers. Then $\Re$ forms an upper semi-continuous closed set decomposition of $S$ ([5], page 99). Define $\phi^{0}: C(T) \rightarrow C(S)$ by the formula

$$
\left(\phi^{0} f\right)(s)=f(\phi(s)), f \in C(T), s \in S .
$$

The map $\phi^{0}$ is an isometric algebra isomorphism of $C(T)$ onto the subalgebra of those functions in $C(S)$ which are constant on each set of $\Re$.

If the closed subalgebra $\phi^{\circ}(C(T))$ is the range of a bounded projection $P$ from $C(S)$, we define the projection constant $p(\phi)$ to be the infimum of $\|P\|$ for all such $P$. We define $p(\phi)=+\infty$ if $\phi^{0}(C(T))$ is uncomplemented. We shall need a result of S. Z. Ditor [2] (formulated here in somewhat different terms) which relates $p(\phi)$ to the topological structure of $S$ and $\phi$. Suppose $\left\{t_{\alpha}\right\}$ is a net in $T$ converging to $t_{0}$. We define the cluster set for $\left\{t_{\alpha}\right\}$ to be

$$
\lim \sup \left\{\phi^{-1}\left(t_{\alpha}\right)\right\}=\left\{\begin{array}{l}
s \mid \text { for each } \alpha_{0} \text { and neighborhood } U \text { of } s, \\
\text { there exists } \alpha \geqq \alpha_{0} \text { with } \phi^{-1}\left(t_{\alpha}\right) \cap U \neq \varnothing
\end{array}\right\} .
$$

The cluster set for $\left\{t_{\alpha}\right\}$ is a nonempty compact subset of the fiber $\phi^{-1}\left(t_{0}\right)$.

Definition 2.1. Let $\phi: S \rightarrow T$ be continuous and onto. Define

$$
M^{(1)}=\left\{\begin{array}{l}
t \mid \text { there exist nets }\left\{t_{\alpha}\right\},\left\{t_{\beta}\right\} \text { converging } \\
\text { to } t \text { whose cluster sets are disjoint }
\end{array}\right\} ;
$$

and inductively define

$$
M^{(n)}=\left\{\begin{array}{l}
t \mid \text { there exist nets }\left\{t_{\alpha}\right\},\left\{t_{\beta}\right\} \text { of points of } M^{(n-1)} \\
\text { converging to } t \text { whose cluster sets are disjoint }
\end{array}\right\} .
$$

Clearly $M^{(1)} \supseteqq M^{(2)} \supseteqq \cdots$. The next theorem is due to S. Z. Ditor [2]. 
THEOREM 2.2. If $M^{(n)} \neq \varnothing$, then $p(\phi) \geqq n$. If $\bigcap_{n=1}^{\infty} M^{(n)} \neq \varnothing$, then $\phi^{0}(C(T))$ is uncomplemented in $C(S)$.

In our application of this result to the Baire classes, matters will be simplified by the fact that the map $\phi: S \rightarrow T$ appearing in that context will be irreducible (i.e., there is no proper closed subset $S_{1}$ of $S$ such that $\left.\phi\left(S_{1}\right)=T\right)$. In the case of irreducible maps the set $M^{(1)}$ is just the set of multiple points for $\phi$. We prove this. The necessary lemma is a consequence of the uppersemicontinuity of $\Re$.

LEMma 2.3. Let $\phi: S \rightarrow T$ be continuous and onto. The following are equivalent.

(1) $\phi$ is irreducible.

(2) For each open set $U \subseteq S$, the set

$$
V=\left\{t \mid \phi^{-1}(t) \subseteq U\right\}
$$

is a nonempty open set in $T$.

(3) For each open set $U$ in $S$, the set

$$
W=U\left\{\phi^{-1}(t) \mid \phi^{-1}(t) \subseteq U\right\}
$$

is an open set dense in $U$.

Proposition 2.4. If $\phi: S \rightarrow T$ is an irreducible onto map, then

$$
M^{(1)}=\left\{t \mid \phi^{-1}(t) \text { contains at least two points }\right\} \text {. }
$$

Proof. Let $s_{1}, s_{2}$ be distinct points of $\phi^{-1}(t)$ with open neighborhoods $U_{1}, U_{2}$ with $\bar{U}_{1} \cap \bar{U}_{2}=\varnothing$. By Lemma $2.3(3)$, there is a net $\left\{t_{\alpha}\right\} \rightarrow t_{0}$ with $\phi^{-1}\left(t_{\alpha}\right) \leqq U_{1}$. Similarly, there is a net $\left\{t_{\beta}\right\} \rightarrow t_{0}$ with $\phi^{-1}\left(t_{\beta}\right) \subseteq U_{2}$. Their cluster sets must be disjoint. Thus every multiple point lies in $M^{(1)}$. The converse inclusion is clear.

3. Nonexistence of complements. Let $\alpha$ and $\beta$ be fixed countable ordinals with $1 \leqq \alpha<\beta$. Then $\mathfrak{B}_{\alpha}$ is a closed subalgebra of $\mathfrak{B}_{\beta}$. Under the representation of $\mathfrak{B}_{\alpha}$ as $C\left(\Omega_{\alpha}\right), \mathfrak{B}_{\beta}$ as $C\left(\Omega_{\beta}\right)$, the natural imbedding of $\mathfrak{B}_{\alpha}$ in $\mathfrak{B}_{\beta}$ induces a canonical continuous onto map $\phi: \Omega_{\beta} \rightarrow \Omega_{\alpha}$. To show that $\mathfrak{B}_{\alpha}$ is uncomplemented in $\mathfrak{B}_{\beta}$ is equivalent to showing that $\phi^{0}\left(C\left(\Omega_{\alpha}\right)\right)$ is uncomplemented in $C\left(\Omega_{\beta}\right)$. We do this by proving that the sets $M^{(n)} \subseteq \Omega_{\alpha}$ for $\phi$ are all nonempty.

Since $\phi$ is one-to-one as a map of $\tau_{\beta}(I)$ onto $\tau_{\alpha}(I)$, and these sets are open and dense in $\Omega_{\beta}$ and $\Omega_{\alpha}$ respectively, it follows from Lemma 2.3 that $\phi$ is irreducible. Thus by Proposition 2.4 $M^{(1)}$ is just the set of multiple points of $\phi$. Since $\mathfrak{B}_{\alpha}$ is a proper subalgebra of $\mathfrak{B}_{\beta}$ 
(see [3]), $M^{(1)}$ is nonempty. We shall prove that if $2 \leqq \alpha<\beta$, then $M^{(1)}=M^{(2)}=\cdots$. The case when $\alpha=1$ is more complicated.

As in Section 1, $K^{\alpha}$ denotes the Boolean algebra of open and closed sets in $\Omega_{\alpha}$. If $A \in K^{\alpha}, \phi^{-1}(A) \in K^{\beta}$, and $\phi^{-1}$ yields an isomorphism of $K^{\alpha}$ onto a subalgebra of $K^{\beta}$. The sets in $\phi^{-1}\left(K^{\alpha}\right)$ are just those sets in $K^{\beta}$ which are unions of fibers for $\phi$.

Definitions 3.1. (a) A set $A \in K^{\beta}$ is of countable or uncountable type depending on whether the Borel set $\tau_{\beta}^{-1}\left(A \cap \tau_{\beta}(A)\right) \in K_{\beta}$ is countable or uncountable.

(b) A point $\omega \in \Omega_{\alpha}$ is a special multiple point for $\phi$ if the fiber $\phi^{-1}(\omega)$ contains at least two distinct points all of whose open and closed neighborhoods are of uncountable type.

We shall prove the following results.

Proposition 3.2. If $1 \leqq \alpha<\beta$, then every set in $K^{\alpha}$ of uncountable type contains a special multiple point for $\phi: \Omega_{\beta} \rightarrow \Omega_{\alpha}$.

THEOREM 3.3. If $1 \leqq \alpha<\beta$, the set $Q$ of special multiple points is nonempty, and

$$
Q \subseteq \bigcap_{n=1}^{\infty} M^{(n)}
$$

THEOREM 3.4. If $1 \leqq \alpha<\beta$, then $\mathfrak{B}_{\alpha}$ is uncomplemented in $\mathfrak{B}_{\beta}{ }^{1}$

Theorems 2.2 and 3.3 clearly imply Theorem 3.4. The role of Proposition 3.2 is to ensure the existence of special multiple points. Its proof is complicated for $\alpha=1$, so we assume it now and prove Theorem 3.3.

Proof of Theorem 3.3. Let $\omega_{0}$ be a special multiple point in $\Omega_{\alpha}$ for $\phi$ and let $\psi_{1}, \psi_{2}$ be distinct points of $\phi^{-1}\left(\omega_{0}\right)$ all of whose $K^{\beta}$ neighborhoods are of uncountable type. Let $B_{1}$ and $B_{2}$ be in $K^{\beta}$ with $\psi_{i} \in B_{i}, i=1,2$ and $B_{1} \cap B_{2}=\varnothing, B_{1} \cup B_{2}=\Omega_{\beta}$. Let $A$ be any $K^{\alpha}$ neighborhood of $\omega_{0}$ in $\Omega_{\alpha}$. The sets

$$
C_{i}=B_{i} \cap \phi^{-1}(A), i=1,2,
$$

are disjoint neighborhoods of $\psi_{i}$ of uncountable type. Consider $C_{1}$. The uncountable Borel set

$$
E_{1}=\tau_{\beta}^{-1}\left(C_{1} \cap \tau_{\beta}(I)\right)
$$

${ }^{1}$ It would suffice to suppose $\beta=\alpha+1$, but this assumption does not simplify the proof. 
contains a Cantor set $F_{1}([6]$, page 446$)$ which is necessarily in $K_{1} \subseteq K_{\alpha}$. Thus the set

$$
D_{1}=\overline{\tau_{\alpha}\left(F_{1}\right)} \subseteq \Omega_{\alpha}
$$

is of uncountable type, $D_{1} \subseteq A$ and $\phi^{-1}\left(D_{1}\right) \subseteq C_{1}$. By Proposition 3.2 $D_{1}$ contains a special multiple point $\omega_{1}$ and clearly $\phi^{-1}\left(\omega_{1}\right) \subseteq C_{1}$. A similar argument applies to $C_{2}$. Since $A$ was arbitrary, it follows that there exist two nets $\left\{\omega_{\xi}\right\},\left\{\omega_{\eta}\right\}$ whose members are special multiple points, such that

$$
\begin{gathered}
\omega_{\xi} \longrightarrow \omega_{0}, \quad \omega_{\eta} \longrightarrow \omega_{0} \\
\phi^{-1}\left(\omega_{\xi}\right) \cong C_{1}, \quad \phi^{-1}\left(\omega_{\eta}\right) \cong C_{2} .
\end{gathered}
$$

Since $C_{1} \cap C_{2}=\varnothing$, the cluster sets $\lim \sup \phi^{-1}\left(\omega_{\xi}\right)$ and $\lim \sup \phi^{-1}\left(\omega_{\eta}\right)$ are nonempty disjoint subsets of $\phi^{-1}\left(\omega_{0}\right)$. Since $\omega_{\xi}, \omega_{\eta} \in M^{(1)}$, by Definition $2.1 \omega_{0} \in M^{(2)}$. However, now each $\omega_{\xi}, \omega_{\eta}$ satisfies the hypotheses for $\omega_{0}$, so by the same argument $\omega_{\xi}, \omega_{\eta} \in M^{(2)}$ and $\omega_{0} \in M^{(3)}$, etc. Thus $\omega_{0} \in \bigcap_{n=1}^{\infty} M^{(n)}$.

To prove Proposition 3.2 we first establish

LEMmA 3.5. Let $1 \leqq \alpha<\beta$. Every set in $K^{\alpha}$ of uncountable type contains a multiple point.

Proof. Let $A$ be a set in $K^{\alpha}$ of uncountable type. The Borel set

$$
E=\tau_{\alpha}^{-1}\left(A \cap \tau_{\alpha}(I)\right)
$$

contains a Cantor set $F$. We may write $F=G \cup H$, where $G$ and $H$ are both uncountable sets lying in $K_{\beta} \sim K_{\alpha}$, since the Cantor set contains Borel subsets of every Baire class relative to itself and hence also relative to $I$ ([3] and [6], page 351). Thus

$$
C=\overline{\tau_{\beta}(G)}, D=\overline{\tau_{\beta}(H)}
$$

are disjoint sets in $K^{\beta} \sim \phi^{-1}\left(K^{\alpha}\right)$. Let $B=\overline{\tau_{\alpha}(F)}$. Then

$$
\phi^{-1}(B)=C \cup D \subseteq \phi^{-1}(A) \text {. }
$$

If $\phi: \phi^{-1}(B) \rightarrow B$ were one-to-one it would be a homeomorphism and $C$ and $D$ would lie in $\phi^{-1}\left(K^{\alpha}\right)$. Thus $B$ (and hence $A$ ) contains a multiple point.

Proposition 3.2 for $\alpha \geqq 2$ now follows from

LEMMA 3.6. Let $2 \leqq \alpha<\beta$ and $\omega_{0}$ be a multiple point for $\phi: \Omega_{\beta} \rightarrow$ $\Omega_{\alpha}$. Each point of $\phi^{-1}\left(\omega_{0}\right)$ has all its $K^{\beta}$ neighborhoods of uncountable type. 
Proof. Let $\psi \in \phi^{-1}\left(\omega_{0}\right)$ and $C$ be a $K^{\beta}$ neighborhood of $\psi$ whose complement also intersects $\phi^{-1}\left(\omega_{0}\right)$. Suppose that

$$
E=\tau_{\beta}^{-1}\left(C \cap \tau_{\beta}(I)\right)
$$

were countable. Then $E \in K_{2} \subseteq K_{\alpha}$ and

$$
C=\phi^{-1} \overline{\left(\tau_{\alpha}(E)\right)},
$$

which is a union of fibers. Since $C$ intersects $\phi^{-1}\left(\omega_{0}\right)$ it must contain it, contradicting the fact $C^{c}$ intersects $\phi^{-1}\left(\omega_{0}\right)$.

COROLLARY 3.7. If $2 \leqq \alpha<\beta$, then every multiple point is a special multiple point, and

$$
Q=M^{(1)}=M^{(2)}=\cdots \text {. }
$$

To prove Proposition 3.2 for $\alpha=1$ we first need

Lemma 3.8. Let $F$ be a Cantor set in $I$. There exists a subset $B$ of $F$ such that $B \Delta C \in K_{2} \sim K_{1}$ for every countable set $C$ in $I_{.}{ }^{2}$

Proof. We start by constructing a certain subset of $I$. Let $A=\bigcup_{n=1}^{\infty} K_{n}$, where each $K_{n}$ is a Cantor set and $A$ is dense in $I$. If $U$ is any nonempty open set in $I, A^{c} \cap U$ is of second category in $U$. Thus $A \cap U$ and $A^{c} \cap U$ are both uncountable. Let $\psi: F \rightarrow I$ be any continuous irreducible onto map. (Such a map exists, since if $\Theta: K \rightarrow I$ is a continuous map from the Cantor set $K$ onto $I$, there exists a closed subset $T$ of $K$ such that $\Theta \mid T$ is irreducible. Since $T$ can have no isolated points, it is homeomorphic to $K$.) Now take $B=\psi^{-1}(A) \subseteq F$. Suppose $V$ is any nonempty relatively open subset of $F$. By Lemma 2.3 there exists a nonempty open set $U$ in $I$ such that $\psi^{-1}(U) \subseteq V$. Thus $B \cap V$ and $B^{c} \cap V$ are both uncountable, since they contain the sets $\psi^{-1}(U \cap A)$ and $\psi^{-1}\left(U \cap A^{c}\right)$.

Now consider $F$ as a subset of $I$ and $B \subseteq F$. Let $C$ be any countable set in $I$. Then $B \Delta C=\left(B \cup D_{1}\right) \cap D_{2}^{c}$, where $D_{1}$ and $D_{2}$ are disjoint countable sets. It $W$ is any open set in $I$ which intersects $F$,

$$
\begin{aligned}
F \cap W \cap(B \Delta C) & \supseteqq W \cap B \cap D_{2}^{c} \\
F \cap W \cap(B \Delta C)^{c} & \supseteqq W \cap B^{c} \cap D_{1}^{c}
\end{aligned}
$$

and these sets are uncountable. Thus the characteristic function of $B \Delta C$ is everywhere discontinuous on $F$, so $B \Delta C \notin K_{1}$ ([6], page 419 ). Since $B$ is an $F_{\sigma}$ set, $B \Delta C \in K_{2}$.

${ }^{2} B \Delta C$ denotes the symmetric difference of $B$ and $C$. 
Proof of Proposition 3.2 when $\alpha=1$. Let $\phi: \Omega_{\beta} \rightarrow \Omega_{1}$ and let $E$ be any set in $K^{1}$ of uncountable type. Since $\tau_{1}^{-1}\left(E \cap \tau_{1}(I)\right)$ contains a Cantor set, it follows from Lemma 3.8 that there exists a set $A \subseteq \phi^{-1}(E) \subseteq \Omega_{\beta}$ (necessarily of uncountable type) such that $A \Delta C \in K^{2} \sim$ $\phi^{-1}\left(K^{1}\right)$ for every set $C$ in $K^{2}$ of countable type. Let $R=\phi(A) \cap \phi\left(A^{c}\right)$. Then $R$ is a compact subset of $E$. We shall prove that $R$ contains a special multiple point for $\phi$. Note that for $\omega \in R, A \cap \phi^{-1}(\omega)$ and $A^{c} \cap \phi^{-1}(\omega)$ are both nonempty. If a point $\omega$ of $R$ is not a special multiple point, then there is at most one point of $\phi^{-1}(\omega)$ all of whose $K^{\beta}$ neighborhoods are of uncountable type. It follows by compactness of $A \cap \phi^{-1}(\omega)$ and $A^{c} \cap \phi^{-1}(\omega)$ that there exists a neighborhood $B_{\omega}$ of $\omega$ in $K^{1}$ such that either $\phi^{-1}\left(B_{\omega}\right) \cap A$ or $\phi^{-1}\left(B_{\omega}\right) \cap A^{c}$ is of countable type in $K^{2}$. Suppose now that $R$ contains no special multiple point. By compactness, a finite number of such neighborhoods $B_{\omega}$ cover $R$. We may suppose them to be disjoint and divide them into two groups whose unions $C_{1}$ and $C_{2}$ satisfy

(1) $C_{1} \cap C_{2}=\varnothing$,

(2) $R \subseteq C_{1} \cup C_{2}$,

(3) $\phi^{-1}\left(C_{1}\right) \cap A$ and $\phi^{-1}\left(C_{2}\right) \cap A^{c}$ are both of countable type.

The open set

$$
W=\left\{\omega \mid \phi^{-1}(\omega) \subseteq A\right\}
$$

is dense in $A$ by Lemma 2.3. Because

$$
\bar{W} \sim W \cong R \cong C_{1} \cup C_{2}
$$

the set

$$
G=W \sim\left(C_{1} \cup C_{2}\right)
$$

is open and closed in $\Omega_{1}$. Moreover

$$
\phi^{-1}(G)=A \sim \phi^{-1}\left(C_{1} \cup C_{2}\right),
$$

because

$$
\phi^{-1}(G)=\phi^{-1}(W) \sim \phi^{-1}\left(C_{1} \cup C_{2}\right) \subseteq A \sim \phi^{-1}\left(C_{1} \cup C_{2}\right) \subseteq \phi^{-1}(G),
$$

since

$$
A \sim \phi^{-1}\left(C_{1} \cup C_{2}\right) \subseteq A \sim \phi^{-1}(R)=\phi^{-1}(W) .
$$

Thus the set $K=A \sim \phi^{-1}(G)$ is a subset of $\phi^{-1}\left(C_{1} \cup C_{2}\right)$, and hence of countable type, and $A \Delta K=\phi^{-1}(G) \in \phi^{-1}\left(K^{1}\right)$. This contradiction proves the existence of a special multiple point for $\phi$ in $R$.

The method above may also be used to prove that $C(I)$ is uncomplemented in $\mathfrak{B}_{\alpha}$ for $\alpha \geqq 1$ and also that each $\mathfrak{B}_{\alpha}$ is uncomplemented in the Banach space $\mathfrak{B}_{\omega_{1}}=\mathbf{U}_{\alpha<\omega_{1}} \mathfrak{B}_{\alpha}$ of all bounded Borel measurable 
functions. We shall not give the details. The fact that $C(I)$ is uncomplemented in $\mathfrak{B}_{\alpha}$ was proved earlier by B. B. Wells, Jr. in [11] using Phillips Lemma ([9], page 525). He also proved that $\mathfrak{B}_{\alpha}$ is uncomplemented in $l_{\infty}(I)$, assuming the continuum hypothesis.

4. Final remarks. We conclude with some observations and open problems.

1. The first observation is that our noncomplementary results for $\mathfrak{B}_{\alpha}$ on $I$ hold equally well when $I$ is replaced by any uncountable compact metric space. If $M$ is such a space, then by a fundamental result of Milutin ([7], [8]) there exists a linear isomorphism $\phi: C(M) \rightarrow$ $C(I)$ of $C(M)$ onto $C(I)$. If $\left\{f_{n}\right\} \subseteq C(M)$, then, by the Lebesgue Dominated Convergence Theorem, $\lim _{n \rightarrow \infty} f_{n}(s)$ exists for each $s \in M$ iff $\lim _{n \rightarrow \infty} \int f_{n} d \mu$ exists for each $\mu \in C(M)^{*}=\phi^{*} C(I)^{*}$ iff $\lim _{n \rightarrow \infty}\left(\phi f_{n}\right)(t)$ exists for each $t \in I$. It follows easily that $\phi$ extends to an isomorphism of $\mathfrak{B}_{1}(M)$ onto $\mathfrak{B}_{1}(I)$ and, inductively, of $\mathfrak{B}_{\alpha}(M)$ onto $\mathfrak{B}_{\alpha}(I), \alpha<\omega_{1}$. Thus $\mathfrak{B}_{\alpha}(M)$ is uncomplemented in $\mathfrak{B}_{\beta}(M)$ for $1 \leqq \alpha<\beta$. Further, F. K. Dashiell has shown (using the Borsuk-Dugundji Theorem) that our results hold in any compact space containing a homeomorph of the Cantor set.

2. A Banach space $X$ is injective if it is complemented in each Banach space $Y$ into which it is imbedded. Theorem 3.4 and the first remark show that none of the Banach spaces $\mathfrak{B}_{\alpha}(M), M$ uncountable compact metric, are injective. This fact may also be proved as a simple consequence of a theorem of $H$. Rosenthal [10]. Suppose $\mathfrak{B}_{\alpha}(M)$ were injective. Since $\mathfrak{B}_{\alpha}(M)$ contains $c_{0}(M)$, it follows from Rosenthal's results that $\mathfrak{B}_{\alpha}(M)$ contains a subspace isomorphic to $l_{\infty}(M)$. However, this subspace has cardinality $2^{c}$, while the cardinality of $\mathfrak{B}_{\alpha}(M)$ is $c$.

The argument just given shows also that $\mathfrak{B}_{\omega_{1}}(M)$, the space of bounded Borel functions on $M$, is not injective. This fact does not seem to follow from our methods.

3. We now give an example of a compact Hausdorff space $\Lambda$ for which $C(\Lambda)$ is complemented in $\mathfrak{B}_{1}(\Lambda)$, even though $C(\Lambda)$ is not injective. We may represent $\mathfrak{B}_{\omega_{1}}(I)=C(\Lambda)$ where $\Lambda$ is a $\sigma$-stonian space. Let $\tau: I \rightarrow \Lambda$ be the natural imbedding of $I$ as an open dense discrete subset of $\Lambda$. Let $g \in \mathfrak{B}_{1}(\Lambda)$ and $\left\{f_{n}\right\}$ be a bounded sequence in $C(\Lambda)$ converging pointwise to $g$ on $\Lambda$. Then the functions $h_{n} \in \mathfrak{B}_{\omega_{1}}(I)$ defined by

$$
h_{n}(t)=f_{n}(\tau(t)), t \in I, n=1,2, \cdots
$$

converge pointwise to a function $k \in \mathfrak{B}_{\omega_{1}}(I)$. Its correspondent $l$ in $C(\Lambda)$ agrees with $g$ on $\tau(I)$ (but not on all of $\Lambda$ unless $g$ is continuous). 
Clearly $l$ is independent of the sequence $\left\{f_{n}\right\}$. The map $g \rightarrow l$ defines a norm one projection of $\mathfrak{B}_{1}(\Lambda)$ onto $C(\Lambda)$.

4. Information concerning the spaces $\Omega_{\alpha}$ is very incomplete.

Question 1. Let $\alpha<\beta$ and $\phi: \Omega_{\beta} \rightarrow \Omega_{\alpha}$ be the canonical map. Is the set of multiple point for $\phi$ closed in $\Omega_{\alpha}$ ?

Question 2. For the map $\phi: \Omega_{1} \rightarrow \Omega_{1}$ can one characterize the special multiple points?

Answers to these questions would greatly simplify the arguments in $\S 3$.

\section{REFERENCES}

1. R. Arens, Representations of *-algebras, Duke Math. J., 14 (1947), 269-282.

2. S. Z. Ditor, Averaging operators in $C(S)$ and lower semi-continuous sections of continuous maps, to appear.

3. R. Engelking, W. Holsztynski and R. Sikorski, Some examples of Borel sets, Colloquium Math., 15 (1966), 271-274.

4. C. Goffman, Real Functions, Boston, 1953.

5. J. L. Kelley, General Topology, Princeton, 1955.

6. K. Kuratowski, Topology, vol. I, New York, 1966.

7. A. A. Milutin, Isomorphism of spaces of continuous functions on compacta having the power of the continuum, Teor. Funkcii Funkcional. Anal. i Prilozen, 2 (1966), 150156 (Russian).

8. A. Pelczynski, Linear extensions, linear averagings, and their applications to linear topological classification of spaces of continuous functions, Dissertationes Math. $\mathbf{5 8}$ (1968).

9. R. S. Phillips, On linear transformations, Trans. Amer. Math. Soc., 48 (1940), 516541.

10. H. Rosenthal, On relatively disjoint families of measures, with some applications to Banach space theory, Studia Math., 27 (1970), 13-36.

11. B. B. Wells, Jr., Uncomplemented subspaces of continuous functions and weak compactness of measures, Thesis, Univ. of California, Berkeley, 1967.

Received October 18, 1971. This research was supported by the National Science Foundation under Grant GP 22712.

UNiversity of CALIfornia, Berkeley 



\section{PACIFIC JOURNAL OF MATHEMATICS}

EDITORS

\author{
H. SAMELSON \\ Stanford University \\ Stanford, California 94305 \\ C. R. HOBBY \\ University of Washington \\ Seattle, Washington 98105
}

\author{
J. DUGUNDJI \\ Department of Mathematics \\ University of Southern California \\ Los Angeles, California 90007 \\ RICHARD ARENS \\ University of California \\ Los Angeles, California 90024
}

\section{ASSOCIATE EDITORS}

E. F. BECKENBACH

B. H. NeUManN

F. WOLF

K. YosHIDA

\section{SUPPORTING INSTITUTIONS}

UNIVERSITY OF BRITISH COLUMBIA

CALIFORNIA INSTITUTE OF TECHNOLOGY

UNIVERSITY OF CALIFORNIA

MONTANA STATE UNIVERSITY

UNIVERSITY OF NEVADA

NEW MEXICO STATE UNIVERSITY

OREGON STATE UNIVERSITY

UNIVERSITY OF OREGON

OSAKA UNIVERSITY
UNIVERSITY OF SOUTHERN CALIFORNIA

STANFORD UNIVERSITY

UNIVERSITY OF TOKYO

UNIVERSITY OF UTAH

WASHINGTON STATE UNIVERSITY UNIVERSITY OF WASHINGTON

AMERICAN MATHEMATICAL SOCIETY NAVAL WEAPONS CENTER 


\section{Pacific Journal of Mathematics}

\section{Vol. 45, No. $1 \quad$ September, 1973}

William George Bade, Complementation problems for the Baire classes .......... 1

Ian Douglas Brown, Representation of finitely generated nilpotent groups ........ 13

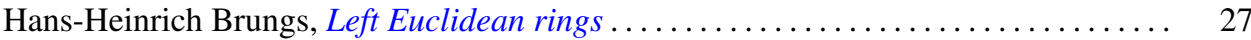

Victor P. Camillo and John Cozzens, A theorem on Noetherian hereditary rings ..... 35

James Cecil Cantrell, Codimension one embeddings of manifolds with locally flat

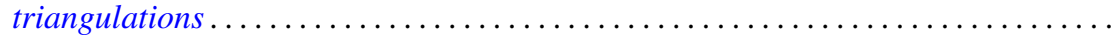

L. Carlitz, Enumeration of up-down permutations by number of rises . . . . . . . . . .

Thomas Ashland Chapman, Surgery and handle straightening in Hilbert cube

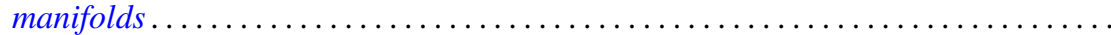

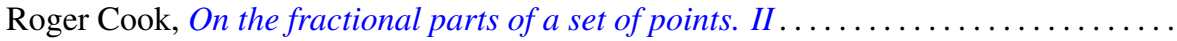

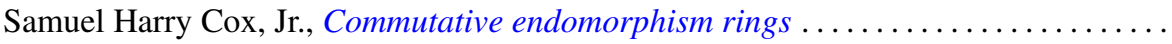

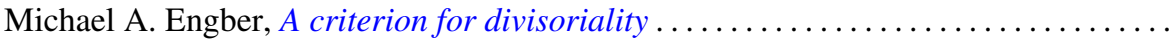

Carl Clifton Faith, When are proper cyclics injective . . . . . . . . . . . . . . 97

David Finkel, Local control and factorization of the focal subgroup . . . . . . . . . 113

Theodore William Gamelin and John Brady Garnett, Bounded approximation by

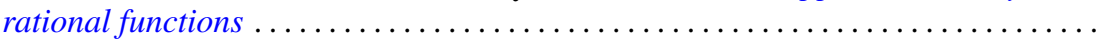

Kazimierz Goebel, On the minimal displacement of points under Lipschitzian

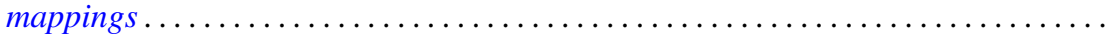

Frederick Paul Greenleaf and Martin Allen Moskowitz, Cyclic vectors for representations associated with positive definite measures: nonseparable

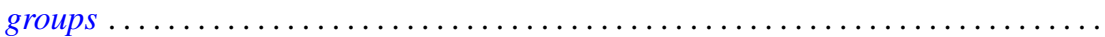

Thomas Guy Hallam and Nelson Onuchic, Asymptotic relations between perturbed linear systems of ordinary differential equations .

David Kent Harrison and Hoyt D. Warner, Infinite primes of fields and completions. .

James Michael Hornell, Divisorial complete intersections . ......

Jan W. Jaworowski, Equivariant extensions of maps ..............

John Jobe, Dendrites, dimension, and the inverse arc function .. .

Gerald William Johnson and David Lee Skoug, Feynman integrals of non-factorable

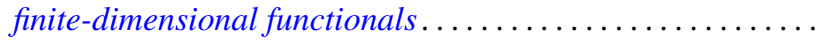

Dong S. Kim, A boundary for the algebras of bounded holomorphic functions ...... 269

Abel Klein, Renormalized products of the generalized free field and its derivatives ... 275

Joseph Michael Lambert, Simultaneous approximation and interpolation in $L_{1}$ and

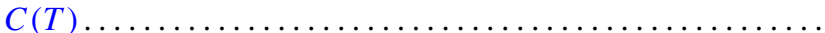

Kelly Denis McKennon, Multipliers of type $(p, p)$ and multipliers of the group

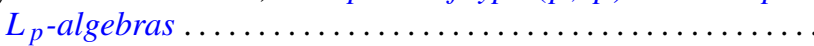

William Charles Nemitz and Thomas Paul Whaley, Varieties of implicative

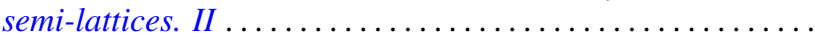

Donald Steven Passman, Some isolated subsets of infinite solvable

Norma Mary Piacun and Li Pi Su, Wallman compactifications on E-completely

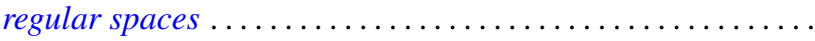

Jack Ray Porter and Charles I. Votaw, $S(\alpha)$ spaces and regular Hausdorff extensions....

Gary Sampson, Two-sided $L_{p}$ estimates of convolution transforms .

Ralph Edwin Showalter, Equations with operators forming a rig
Raymond Earl Smithson, Fixed points in partially ordered sets .

Victor Snaith and John James Ucci, Three remarks on symmetric products and

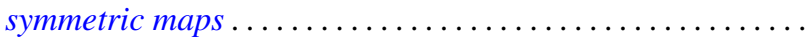

